\title{
A Weak Signal Detection Application Based on Hyperchaotic Lorenz System
}

\author{
Abdullah GOKYILDIRIM, Yilmaz UYAROGLU, Ihsan PEHLIVAN
}

\begin{abstract}
Due to accurate capability to detect weak signal, chaotic oscillators have become an interesting topic for many scientific researches. In this paper, two hyperchaotic Lorenz systems are presented to detect weak signal. These systems are chosen because of their parametric variety and high applicability. Dynamic behaviors of two hyperchaotic systems are analysed in detail. For this purpose, the Lyapunov exponent values and bifurcation behaviours of two hyperchaotic systems are analysed for weak signal detection applications. The relationship between the system state and the amplitude of the weak signal is defined by examining the Lyapunov exponents of the system. So, dynamic characteristics of two chaotic oscillators are observed by this way. The critical chaotic parametric threshold value of a chaotic system is easily determined by the bifurcation analysis. The bifurcation threshold value named as tangent bifurcation point is the most suitable one to detect weak signal. For this purpose, the tangent bifurcation points of these systems are determined via bifurcation analysis. Additionally, weak signal detection applications of two hyperchaotic systems are also studied. The applicability of the proposed systems is shown by these applications. These systems also detect the weak signal with low signal to noise ratio (SNR). Simulation results obtained from Matlab-Simulink ${ }^{\circledR}$ program verify the studied method.
\end{abstract}

Keywords: bifurcation; chaotic systems; hyperchaos; Lyapunov method; signal to noise ratio; weak signal detection

\section{INTRODUCTION}

Chaos theory explains the complicated behaviours of a nonlinear system. Chaotic systems are seemingly random and depend on the initial conditions sensitively. These systems have sensitive dependence on the internal variations of initial conditions. Besides this, they are immune to background noise. Because of these features of the chaotic systems, this theory has become a hot research topic. In 1963, Lorenz presented a new chaotic system which consists of nonlinear differential equations known as the Lorenz attractor [1]. Since then, the phenomenon of chaos has become an attractive research topic on many chaos-based technologies and information systems [2-11].

In 1992, Birx showed that chaotic systems are sensitive to weak signal but immune to the noise [12]. In the last two decades, many studies have been made on signal detection [13-23]. Li and Yang used the modified Duffing-Holmes equation to detect $n V$ level signal [24]. Chunyan and Yaowu presented the Melnikov method to observe the threshold value of oscillator and used crosscorrelation method to get lower SNR threshold [25]. But the precision of threshold value is low at Melnikov method. Furthermore Melnikov function is very complex [26]. On the other hand, many researchers determined the threshold value of a chaotic system via Lyapunov exponents method [27, 28]. These studies showed that Lyapunov method increased the detection accuracy.

Additionally the hyperchaotic systems have become a focal research topic. Very recently, many studies have been made on hyperchaotic attractors and their dynamical behaviours [29, 30].

This paper is organized as follows: in section 2 Basic principles of Lorenz System and two hyperchaotic Lorenz systems are examined. The relationship between the system state and the amplitude of the forcing term is defined via Lyapunov exponents of the studied systems. Bifurcation analysis easily determines these systems' critical tangent bifurcation points. This critical point is the most suitable one to detect weak signal. For this aim, the tangent bifurcation points of these systems are determined by bifurcation analysis.
In Section 3, weak signal detection based on chaos theory is presented. Detection result comparisons of the studied systems are given in this section. Summarized simulation results show that this method is simple and effective. Section 4 contains the conclusions.

\section{BASIC PRINCIPLES OF SINUSOIDAL FORCED LORENZ SYSTEM}

\subsection{Traditional Lorenz System}

Traditional Lorenz chaotic system's dynamical model is as follows:

$$
\left\{\begin{array}{l}
\dot{x}=a(y-x) \\
\dot{y}=c x-x z+y \\
\dot{z}=x y-b z
\end{array}\right.
$$

where $x, y$ and $z$ are state variables. When $a=10, b=8 / 3$ and $c=25$, the system shows chaotic behaviour. The chaotic phase portraits are shown in Fig. 1.
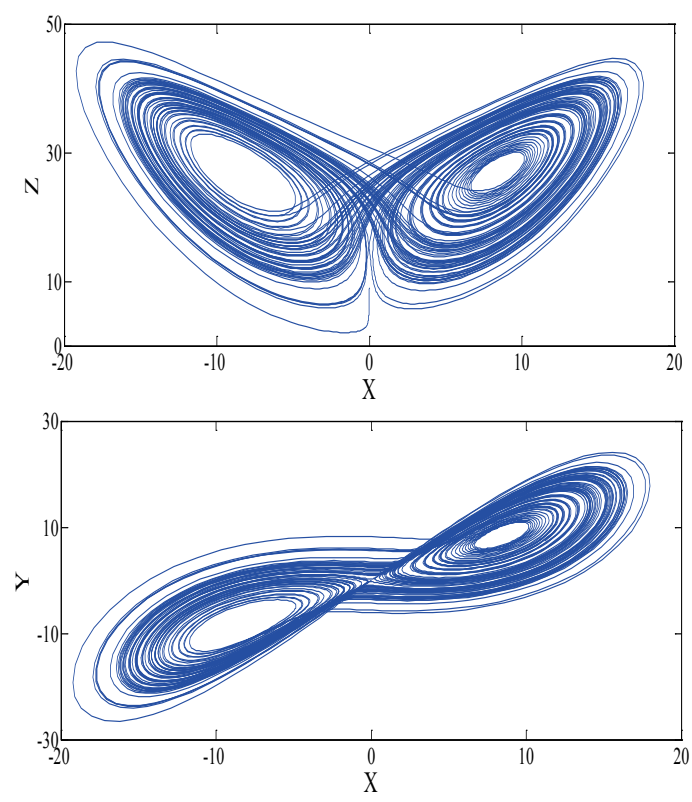

Figure 1 Chaotic phase portraits of Lorenz system with $a=10, b=8 / 3$ and $c=25$ $\left(x_{0}, y_{0}, z_{0}=0,-0.01,9\right)$ 


\subsection{Generation of Four-Dimensional Hyperchaotic Sinusoidal Forced System-A}

The simplified Lorenz system-A is described as follows [31]:

$$
\left\{\begin{array}{l}
\dot{x}=10(y-x) \\
\dot{y}=(24-4 c) x-x z+c y \\
\dot{z}=x y-8 z / 3
\end{array}\right.
$$

where $\mathrm{c}$ is an adjustable parameter. Then we define a new variable which is a simple sinusoidal control function $c=c_{0} \sin (\omega t)$. The new function $c=c_{0} \sin (\omega t)$ is a timevarying forcing term. This function transforms the threedimensional autonomous system (2) into a threedimensional non-autonomous system, which is equivalent to a four-dimensional autonomous system:

$$
\left\{\begin{array}{l}
\dot{x}=10(y-x) \\
\dot{y}=\left(24-4 c_{0} \sin (u)\right) x-x z+c_{0} \sin (u) y \\
\dot{z}=x y-8 z / 3 \\
\dot{u}=\omega
\end{array}\right.
$$

where $\omega$ is the angular frequency of periodic driving power and $c_{0}$ is the amplitude. If we fix $\omega$ then as $c_{0}$ varies from small to big, the system states may appear like a fixed point, a torus, a chaotic motion or a hyperchaotic motion.

\subsection{Generation of Four-Dimensional Hyperchaotic Sinusoidal Forced System-B}

If a simple sinusoidal control function $c=c_{0} \sin (\omega t)$ is added to the second equation of Lorenz system (1), a non-autonomous system is obtained as follows [32]:

$\left\{\begin{array}{l}\dot{x}=10(y-x) \\ \dot{y}=25 x-x z+y+c_{0} \sin (\omega t) \\ \dot{z}=x y-8 z / 3\end{array}\right.$

This non-autonomous system is equivalent to a fourdimensional autonomous system:

$$
\left\{\begin{array}{l}
\dot{x}=10(y-x) \\
\dot{y}=25 x-x z+y+c_{0} \sin (u) \\
\dot{z}=x y-8 z / 3 \\
\dot{u}=\omega
\end{array}\right.
$$

According to Eqs. (3) and (5), the structures of the sinusoidal forced Lorenz system-A and system-B are apparently more complex than the original system.

\subsection{Lyapunov Exponents and Bifurcation Behaviour}

The Lyapunov exponents (LE) examine the dynamic characteristics of the dynamic system. They are parameters used to make a decision about a nonlinear chaotic system. Therefore, these exponents help to judge whether a chaotic system is in chaos state or not $[33,34]$. Poincare map of a four-dimensional non-autonomous system is:

$\left\{\begin{array}{l}x_{n+1}=f_{1}\left(x_{n}, y_{n}, z_{n}, u_{n}\right) \\ y_{n+1}=f_{2}\left(x_{n}, y_{n}, z_{n}, u_{n}\right) \\ z_{n+1}=f_{3}\left(x_{n}, y_{n}, z_{n}, u_{n}\right) \\ u_{n+1}=f_{4}\left(x_{n}, y_{n}, z_{n}, u_{n}\right)\end{array}\right.$

Its Jacobian matrix:

$$
\boldsymbol{J}\left(x_{n}, y_{n}, z_{n}, u_{n}\right)=\left[\begin{array}{llll}
\frac{d f_{1}}{d x_{n}}, & \frac{d f_{1}}{d y_{n}}, & \frac{d f_{1}}{d z_{n}}, & \frac{d f_{1}}{d u_{n}} \\
\frac{d f_{2}}{d x_{n}}, & \frac{d f_{2}}{d y_{n}}, & \frac{d f_{2}}{d z_{n}}, & \frac{d f_{2}}{d u_{n}} \\
\frac{d f_{3}}{d x_{n}}, & \frac{d f_{3}}{d y_{n}}, & \frac{d f_{3}}{d z_{n}}, & \frac{d f_{3}}{d u_{n}} \\
\frac{d f_{4}}{d x_{n}}, & \frac{d f_{4}}{d y_{n}}, & \frac{d f_{4}}{d z_{n}}, & \frac{d f_{4}}{d u_{n}}
\end{array}\right]
$$

For the purpose of calculating Lyapunov exponents, Jacobian matrices of System-A and System-B in Eqs. (3) and (5) must be calculated. Jacobian matrices for SystemA and System-B are respectively as follows:

$\boldsymbol{J}_{A}=\left[\begin{array}{cccc}-10 & 10 & 0 & 0 \\ \left(-4 c_{0} \sin (\omega t)\right. & c_{0} \sin (\omega t) & -x & \left(-4 c_{0} \omega \cos (\omega t) x\right. \\ -z+24) & & & \left.+c_{0} \omega \cos (\omega t) y\right) \\ y & x & -8 / 3 & 0 \\ 0 & 0 & 0 & 0\end{array}\right]$

$\boldsymbol{J}_{B}=\left[\begin{array}{cccc}-10 & 10 & 0 & 0 \\ 25-z & 1 & -x & \omega c_{0} \cos (\omega t) \\ y & x & -8 / 3 & 0 \\ 0 & 0 & 0 & 0\end{array}\right]$

Assuming the point consecutive mapping from the initial point $P_{0}\left(x_{0}, y_{0}, z_{0}, u_{0}\right)$ to $P_{1}\left(x_{1}, y_{1}, z_{1}, u_{1}\right), P_{2}\left(x_{2}, y_{2}, z_{2}, u_{2}\right)$, $\ldots, P_{n}\left(x_{n}, y_{n}, z_{n}, u_{n}\right)$, the Jacobian matrix of previous $(n-1)^{t h}$ point is:

$$
\begin{gathered}
\boldsymbol{J}_{0}=\boldsymbol{J}\left(x_{0}, y_{0}, z_{0}, u_{0}\right) \\
\boldsymbol{J}_{1}=\boldsymbol{J}\left(x_{1}, y_{1}, z_{1}, u_{1}\right) \\
\vdots \\
\boldsymbol{J}_{n-1}=\boldsymbol{J}\left(x_{n-1}, y_{n-1}, z_{n-1}, u_{n-1}\right)
\end{gathered}
$$

Define $J^{(n)}=J_{n-1} J_{n-2} \ldots J_{1} J_{2}$. The modulo of eigen-value for $J^{(n)}$ is $J_{1}^{(n)}, J_{2}^{(n)}, J_{3}^{(n)}$ and $J_{4}^{(n)}\left(J_{1}^{(n)}>J_{2}^{(n)}>J_{3}^{(n)}>J_{4}^{(n)}\right)$.

The Lyapunov exponents can be written in the following equation: 


$$
\begin{aligned}
& \lambda_{1}=\lim _{n \rightarrow \infty} \sqrt[n]{J_{1}^{(n)}} \\
& \lambda_{2}=\lim _{n \rightarrow \infty} \sqrt[n]{J_{2}^{(n)}} \\
& \lambda_{3}=\lim _{n \rightarrow \infty} \sqrt[n]{J_{3}^{(n)}} \\
& \lambda_{4}=\lim _{n \rightarrow \infty} \sqrt[n]{J_{4}^{(n)}}
\end{aligned}
$$

For a four-dimensional system, when all four exponents are negative, the system begins to converge to a fixed point. When the Lyapunov distribution is $(0,-,-,-)$ and $(+, 0,-,-)$, the system state seems like a limit cycle and a strange attractor respectively [31].

When the system is in chaotic state, there are four exponents. The first exponent's sign is positive, the second exponent's sign is zero, the third exponent's sign is negative and the fourth exponent's sign is negative. If the first exponent shows a rapid decrease to negative value, the system state seems like a large scale periodic state. If the Lyapunov distribution is $(+,+, 0,-)$, a hyperchaotic motion appears. Tab. 1 shows the relationship between the Lyapunov exponents and the system's state.

Table 1 Lyapunov exponents and system's state of a four dimensional system

\begin{tabular}{|c|c|}
\hline $\begin{array}{c}\text { Signs of Lyapunov exponents } \\
\left(\lambda_{1}, \lambda_{2}, \lambda_{3}, \lambda_{4}\right)\end{array}$ & System's state \\
\hline$(-,-,-,-)$ & Fixed point \\
\hline$(0,-,-,-)$ & Limit cycle \\
\hline$(+, 0,-,-)$ & Strange attractor \\
\hline$(+,+, 0,-)$ & Hyperchaotic \\
\hline
\end{tabular}

Complex dynamical behaviours of a chaotic system can be easily obtained by examining the bifurcation behaviour [35, 36]. Bifurcation analyses in this work show that the studied sinusoidal forced Lorenz systems exhibit different system states such as limit cycles, chaos, tangent bifurcation and great scale periodic.

The threshold value of the system can be obtained by determining the tangent bifurcation point. Detection of this bifurcation threshold value is required to detect weak signal. In this way, the detection process is eased.

\subsection{Lyapunov Exponents and Tangent Bifurcation Analysis of Sinusoidal Forced Lorenz System-A and System-B}

In this section, the dynamical behaviour of the chaotic sinusoidal forced Lorenz system is analysed via Lyapunov exponents and bifurcation diagrams.

Case-A: Lyapunov exponents and the corresponding bifurcation diagram of the local maximum of $x$ versus $c$ are shown in Fig. 2. A tangent bifurcation exists in periodic window when $c=0.991 . x$ is selected for visualizing the tangent bifurcation point.

Chaotic attractors of the system-A with $\omega=10$ are shown in Fig. 3. When parameter $c$ changes from 0.991 to 0.992 , system state turns to great scale periodic state.

Case-B: Lyapunov exponents and the corresponding bifurcation diagram of the local maximum of $x$ versus $\omega$ are shown in Fig. 4. A tangent bifurcation exists in periodic window when $\omega=10.017$.

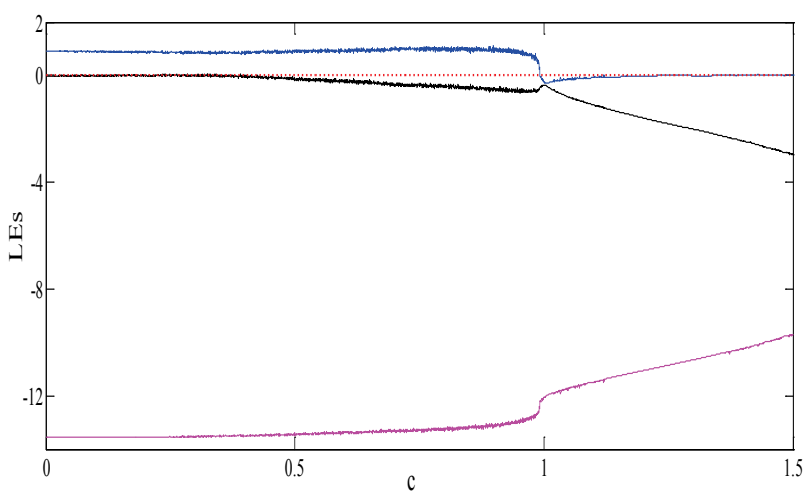

(a)

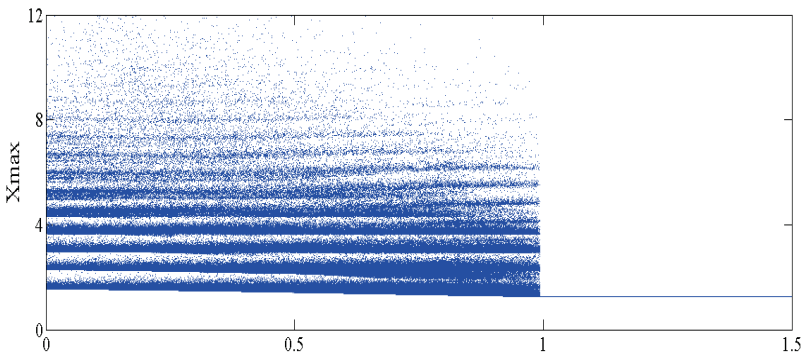

(b)

Figure 2 Lyapunov exponents and bifurcation diagram of the system-A with $\omega=10$ (a) Lyapunov exponents versus ${ }^{c}$ (b) Bifurcation diagram

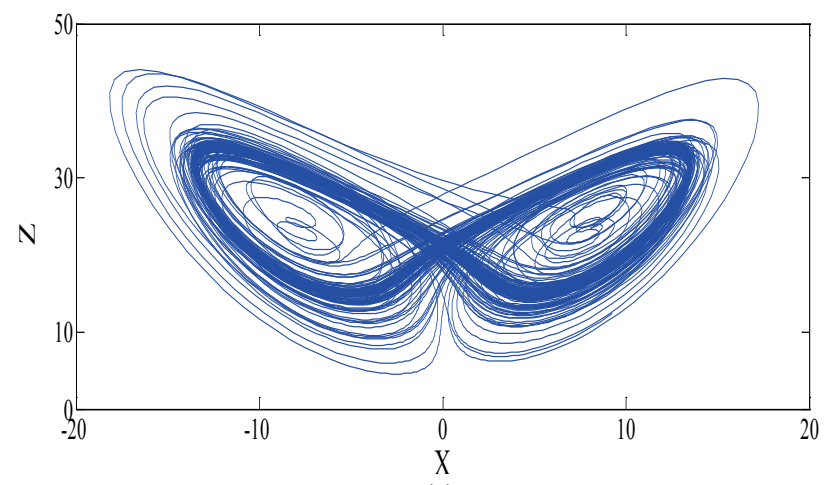

(a)

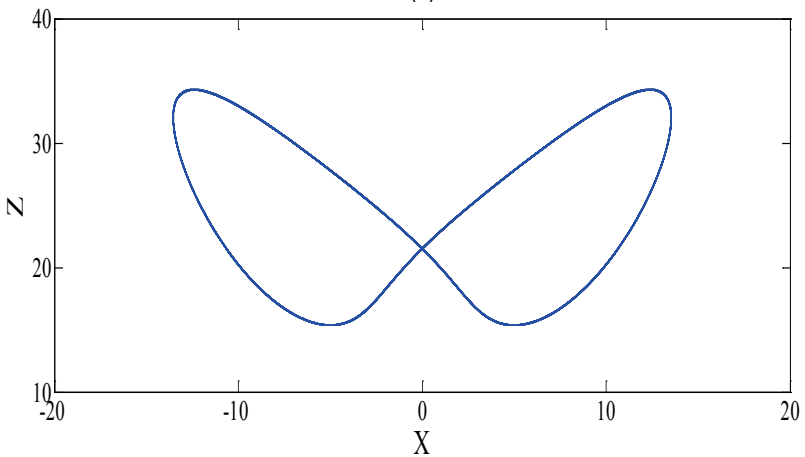

(b)

Figure 3 Chaotic phase portraits of the system-A ( $\omega=10, t=50-500 \mathrm{sn})$

$$
\text { (a) } c=0.991 \text { (b) } c=0.992
$$

Chaotic attractors of the system-A with $c=1$ are shown in Fig. 5. When parameter $\omega$ changes from 10.017 to 10.016 , system state changes from chaotic critical state to great scale periodic state.

Case-C: Lyapunov exponents and the corresponding bifurcation diagram of the local maximum of $x$ versus $c$ are shown in Fig. 6. A tangent bifurcation exists in periodic window when $c=33.771$. 


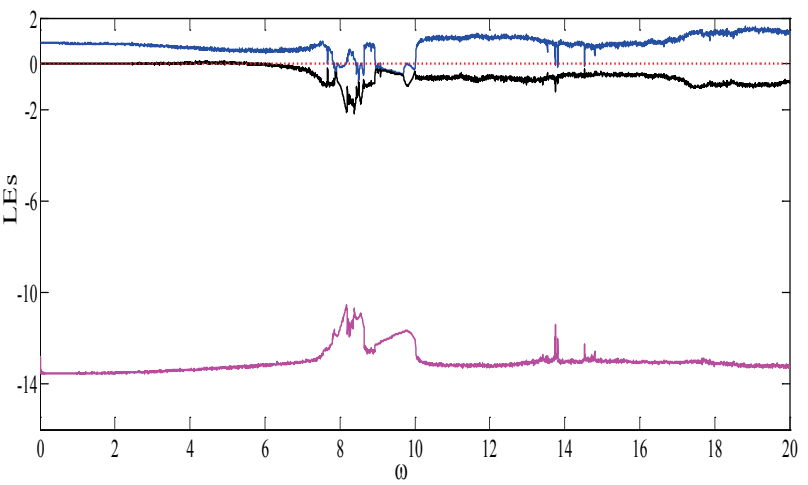

(a)

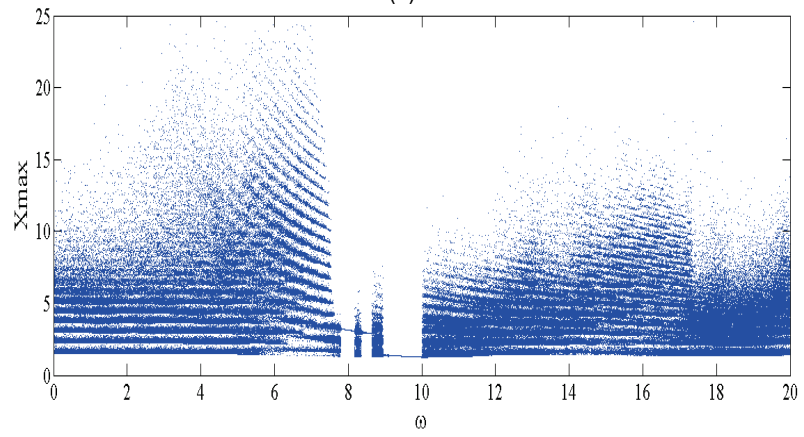

(b)

Figure 4 Lyapunov exponents and bifurcation diagram of the system-A with $c=1$ (a) Lyapunov exponents versus ${ }^{\omega}$ (b) Bifurcation diagram

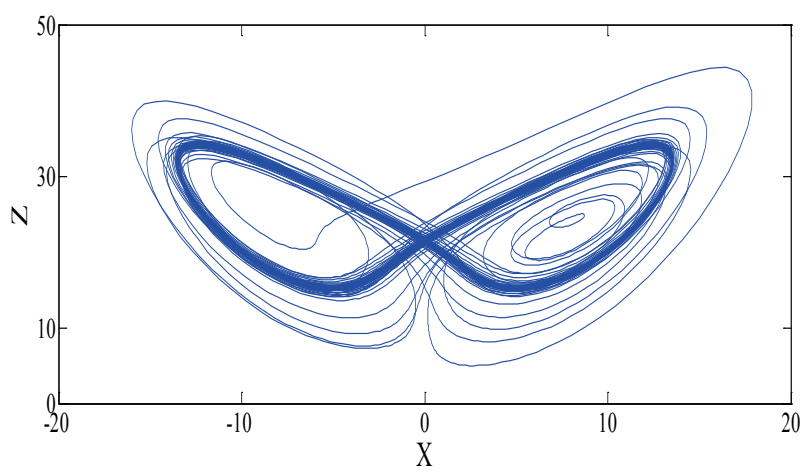

(a)

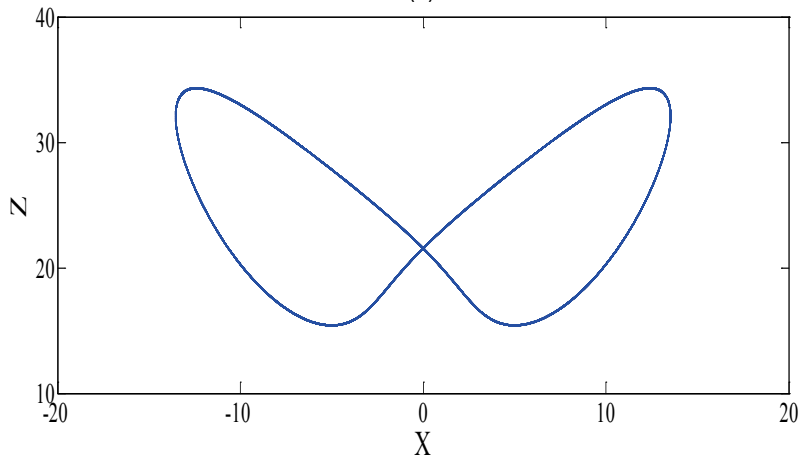

(b)

Figure 5 Chaotic phase portraits of the system-A $(c=1, t=50-500 \mathrm{sn})$ (a) $\omega=10.017$ (b) $\omega=10.016$

Chaotic attractors of the system-B with $\omega=9.6$ are shown in Fig. 7. When parameter $c$ changes from 33.771 to 33.772 , system state turns to great scale periodic state.

Case-D: Lyapunov exponents and the corresponding bifurcation diagram of the local maximum of $x$ versus $\omega$ are shown in Fig. 8. A tangent bifurcation exists in periodic window when $\omega=6.430$.

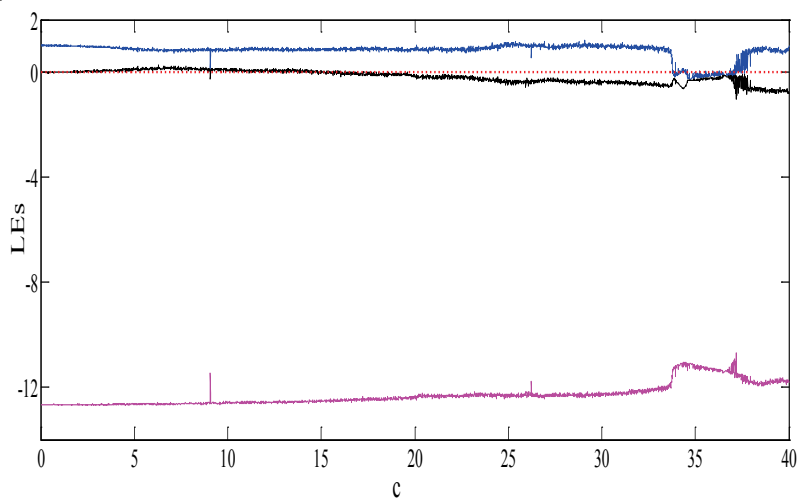

(a)

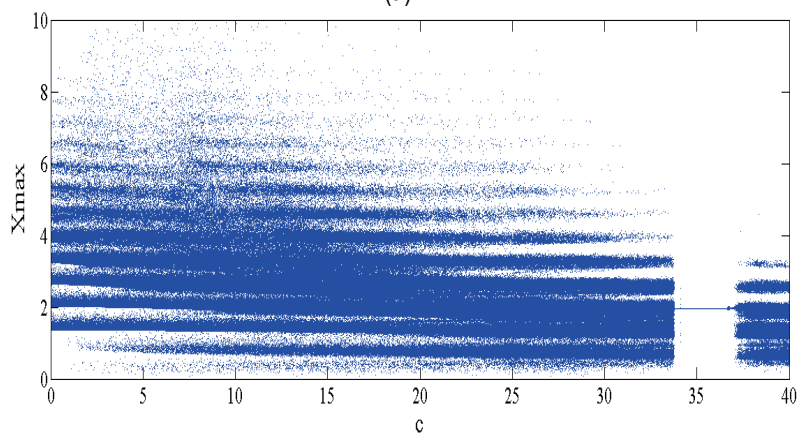

(b)

Figure 6 Lyapunov exponents and bifurcation diagram of the system-B with $\omega=9.6$ (a) Lyapunov exponents versus ${ }^{c}$ (b) Bifurcation diagram

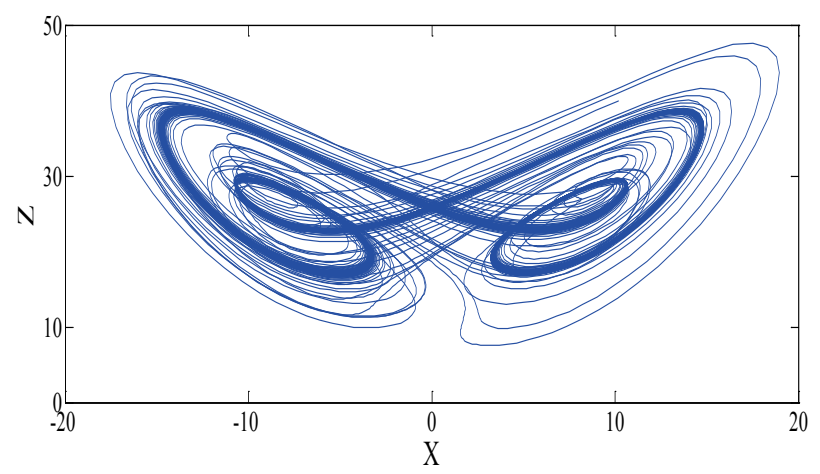

(a)

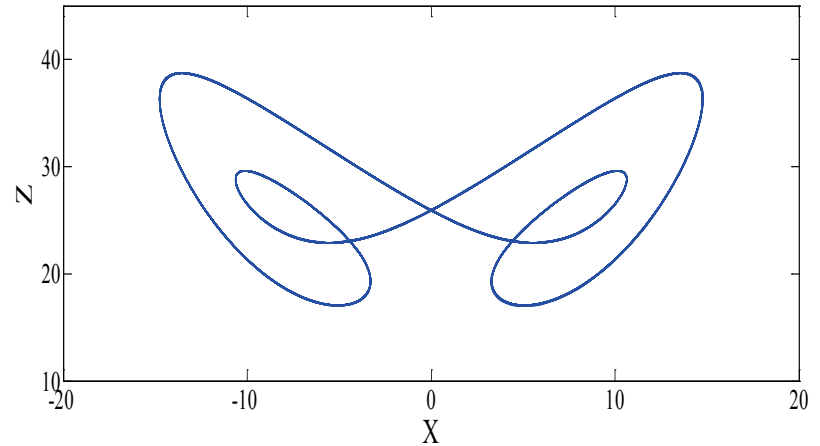

(b)

Figure 7 Chaotic phase portraits of the system-B $(\omega=9.6$,

$$
t=50-500 \mathrm{sn})(\mathrm{a})^{c}=33.771 \text { (b) } c=33.772
$$

Chaotic attractors of the system-B with $c=35$ are shown in Fig. 9. When parameter $\omega$ changes from 6.430 to 6.429 , system state turns to great scale periodic state. 


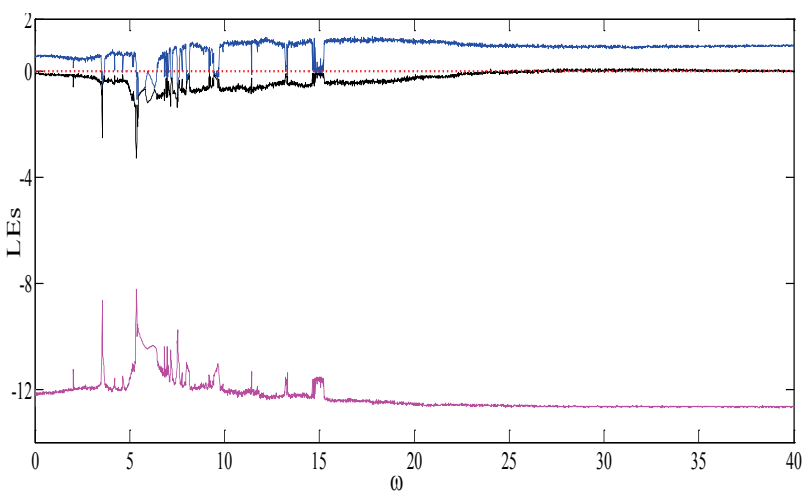

(a)

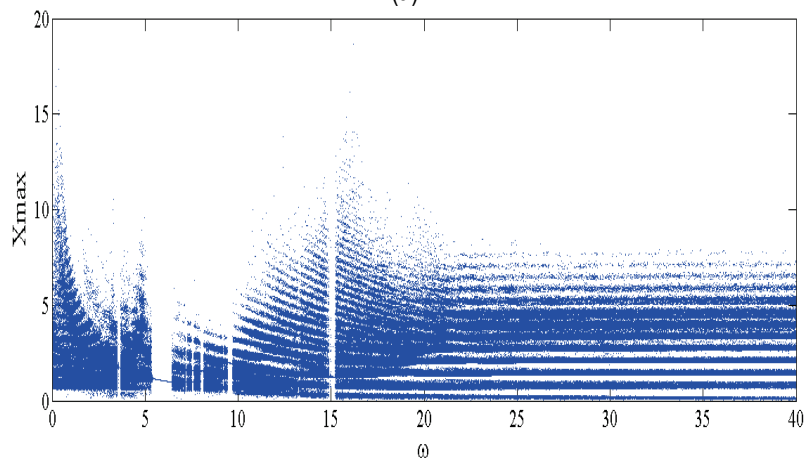

(b)

Figure 8 Lyapunov exponents and bifurcation diagram of the system-B with $c=35$ (a) Lyapunov exponents versus ${ }^{\omega}$ (b) Bifurcation diagram

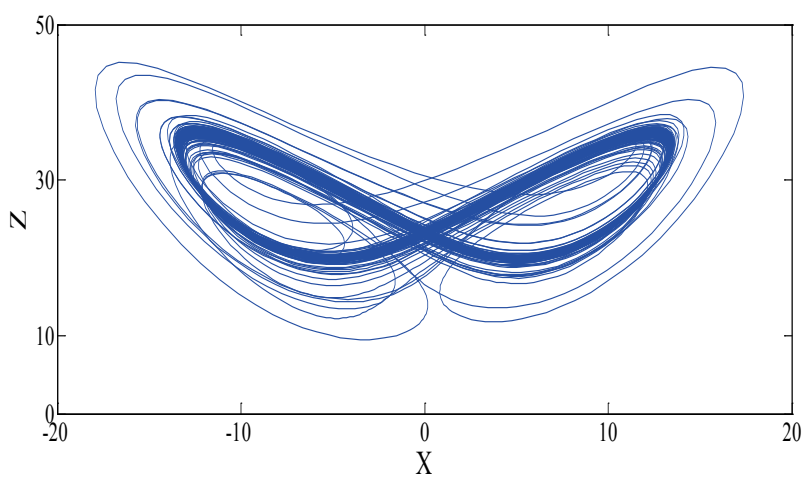

(a)

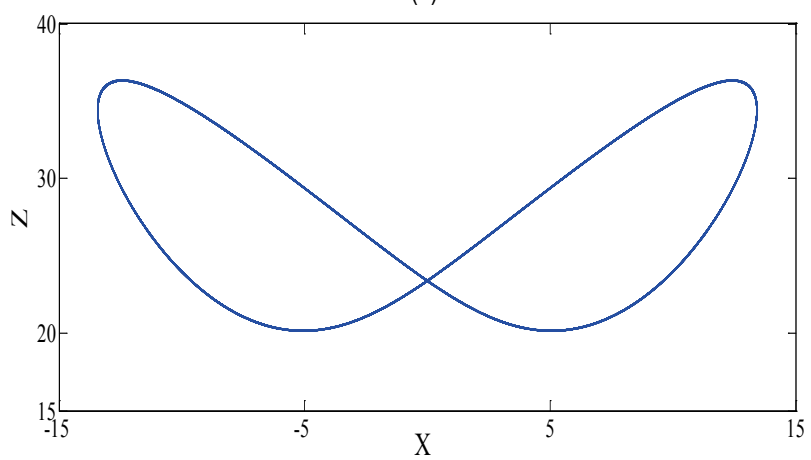

(b)

Figure 9 Chaotic phase portraits of the system-B $(c=35$, $t=50-500 s n)(\mathrm{a}) \omega=6.430$ (b) $\omega=6.429$

\section{WEAK SIGNAL DETECTION BASED ON CHAOS THEORY \\ 3.1 Weak Signal Detection Principle}

Chaotic system state rapidly changes from chaotic critical state to great scale periodic state on the tangent bifurcation point. This critical point is used by detection system to detect weak signal easily. In order to detect weak signal by system-A, an input which includes weak signal and noise is added to Eq. (3).

$$
\left\{\begin{array}{l}
\dot{x}=10(y-x) \\
\dot{y}=\left(24-4\left(c_{0} \sin (u)+\text { input }\right)\right) x-x z+\left(c_{0} \sin (u)+\text { input }\right) y \\
\dot{z}=x y-8 z / 3 \\
\dot{u}=\omega
\end{array}\right.
$$

The same is valid for system-B in which an input which includes weak signal and noise is added to Eq. (5).

$$
\left\{\begin{array}{l}
\dot{x}=10(y-x) \\
\dot{y}=25 x-x z+y+c_{0} \sin (u)+\text { input } \\
\dot{z}=x y-8 z / 3 \\
\dot{u}=\omega
\end{array}\right.
$$

where input is:

$$
\text { input }=s(\tau)+n(\tau)=r \sin (\omega \tau)+n(\tau)
$$

$r \sin (\omega \tau)$ is weak signal and $n(\tau)$ is the Gaussian white noise in Eq. (12).

\subsection{Signal to Noise Ratio Analysis}

The signal to noise ratio $(S N R)$ is defined as ratio between the average power of the weak signal and noise [15]. The input $S N R$ can be written in the following equation:

$$
(S N R)_{i}=\left.\frac{H_{S}(\omega)}{H_{n}(\omega)}\right|_{\omega=\omega_{0}}
$$

$H_{s}(\omega)$ is the power spectrum amplitude of the weak signal and $H_{n}(\omega)$ is the power spectrum amplitude of noise. These can be decomposed as:

$$
\begin{aligned}
& H_{s}(\omega)=\left\{\begin{array}{l}
\frac{r^{2}}{4}, \omega= \pm \omega_{0} \\
=0, \omega \neq \pm \omega_{0}
\end{array}\right. \\
& H_{n}(\omega)=\int_{-\infty}^{+\infty} P_{n}^{2} \delta(\tau) e^{-j \omega \tau} d \tau=P_{n}^{2}
\end{aligned}
$$

Eq. (13) can be expressed as:

$$
(S N R)_{i}=\frac{r^{2}}{4 P_{n}^{2}}
$$

where $P_{n}$ is noise power and $r$ is amplitude of the weak signal. Eq. (16) can be written in the logarithm form: 
$(S N R)_{i}=10 \lg \frac{0.5 \times r^{2}}{P_{n}}(d B)$

\subsection{Simulations and Results}

The simulated models are constructed by using Matlab-Simulink $^{\circledR}$ software. The fourth-order RungaKutta algorithm is used to solve two Lorenz systems. The diagrams of chaotic detection systems are shown in Fig. 10 and Fig. 11 respectively. They include signal source, integrator, gain and function. Simulation time $t$ is $150-1500$ sn .

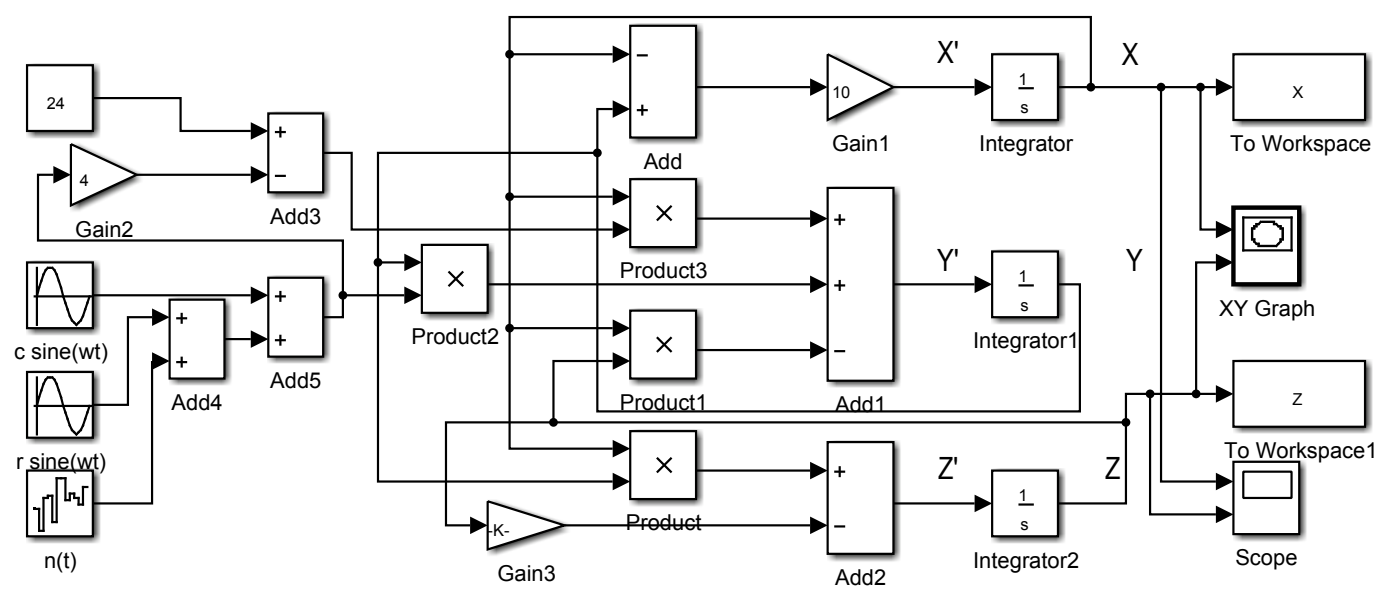

Figure 10 Simulink diagram of the chaotic detection system-A

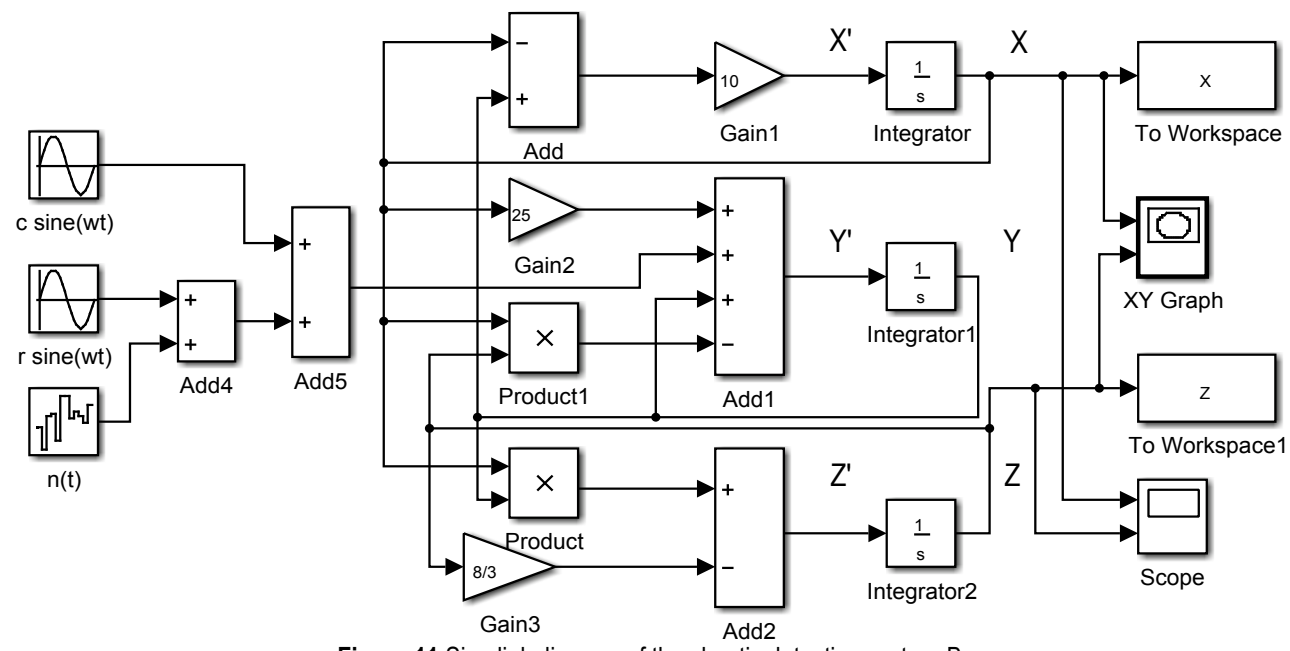

Figure 11 Simulink diagram of the chaotic detection system-B

Table 2 Comparison of detection results

\begin{tabular}{|c|c|c|c|c|c|}
\hline & $\begin{array}{r}\text { Weak signal } \\
\text { amplitude }(r)\end{array}$ & $\begin{array}{c}\text { Threshold } c \text { to detect weak } \\
\text { signal }\end{array}$ & Noise power $\left(P_{n}\right)$ & Noise effect & $\operatorname{SNR}(d B)$ \\
\hline \multirow[b]{2}{*}{ System-A } & $10^{-3}$ & 1.002 & $10^{-3}$ & $11 \times 10^{-3}$ & -33 \\
\hline & $10^{-4}$ & 0.9968 & $10^{-4}$ & $5 \times 10^{-4}$ & -43 \\
\hline \multirow{3}{*}{$(\omega=10$ and } & $10^{-5}$ & 0.99273 & $10^{-5}$ & $8.7 \times 10^{-4}$ & -53 \\
\hline & $10^{-6}$ & 0.992027 & $10^{-6}$ & $16 \times 10^{-5}$ & -63 \\
\hline & $10^{-6}$ & 0.992871 & $10^{-5}$ & $10.03 \times 10^{-4}$ & -73 \\
\hline$\left.c_{0}=0.991868\right)$ & $10^{-6}$ & 0.998504 & $10^{-4}$ & $6.637 \times 10^{-3}$ & -83 \\
\hline \multirow[b]{2}{*}{ System-B } & $10^{-3}$ & 33.778 & $10^{-3}$ & $7 \times 10^{-3}$ & -33 \\
\hline & $10^{-4}$ & 33.7729 & $10^{-4}$ & $12 \times 10^{-4}$ & -43 \\
\hline \multirow{3}{*}{$(\omega=9.6$ and } & $10^{-5}$ & 33.77181 & $10^{-5}$ & $11 \times 10^{-5}$ & -53 \\
\hline & $10^{-6}$ & 33.771722 & $10^{-6}$ & $2.2 \times 10^{-5}$ & -63 \\
\hline & $10^{-6}$ & 33.771793 & $10^{-5}$ & $9.3 \times 10^{-5}$ & -73 \\
\hline$\left.c_{0}=33.771700\right)$ & $10^{-6}$ & 33.773103 & $10^{-4}$ & $14.03 \times 10^{-4}$ & -83 \\
\hline
\end{tabular}

The detection application is explained in the following steps:

Step-1 Put the noise into the system, when the system is in chaotic state.
Step-2 Observe the value of parameter $c$. If the value of $c$ is on the first tangent bifurcation point, the system state will change from chaotic critical state to great scale periodic state. Note this value of $c$. 
Step-3 Put the weak signal into the system. Observe the value of parameter $c$. If the value of $c$ is on the second tangent bifurcation point, the system state will change from chaotic critical state to great scale periodic state. Note this second tangent bifurcation point. Weak signal amplitude is equal to the difference between two tangent bifurcation points. Tab. 2 shows comparison of detection results.

As can be seen in Tab. 2, the experiment results demonstrated the relation between noise power and noise effect. It shows that when the value of $\omega$ is 10 in the system-A, the weak signal detecting accuracy is high. Additionally, when the value of $\omega$ is 9.6 in the system-B, the weak signal detecting accuracy is also high. The simulation result shows that these two systems have high weak signal detection accuracy. Finally, $S N R$ analysis is studied. In order to Eq. (17) the smallest reachable $S N R$ threshold is:

$S N R=10 \lg \frac{0.5 \times\left(10^{-6}\right)^{2}}{\left(10^{-4}\right)^{2}}=-83.0103(\mathrm{~dB})$

\section{CONCLUSION}

In this paper, we proposed two hyperchaotic versions of the Lorenz model called sinusoidal forced Lorenz system-A and system-B to detect weak signal. For this aim, the Lyapunov exponent values and bifurcation behaviours of two hyperchaotic systems are analysed for weak signal detection applications. The relationship between the system state and the amplitude of the weak signal is defined by examining the Lyapunov exponents of the system. So, dynamic characteristics of two chaotic oscillators are observed in this way. Additionally, a chaotic system's complex dynamical behaviour is easily determined by bifurcation analysis. The tangent bifurcation point is the most suitable one to detect weak signal. For this purpose, the tangent bifurcation points of the studied systems are determined via bifurcation analysis.

Finally, weak signal detection applications of two hyperchaotic Lorenz systems are studied. The chaotic detecting systems' SNR analysis is studied too. Simulation block diagrams of chaotic detection systems are designed in Matlab-Simulink ${ }^{\circledR}$.

The studied systems' dynamic analyses show that these systems have not only parametric variety but also high applicability. Additionally, simulation results indicate that these two hyperchaotic systems can detect weak signal with high detection accuracy and low SNR. Further study should be done on weak signal detection application with lower SNR.

\section{REFERENCES}

[1] Lorenz, E. N. (1963). Deterministic Non-Periodic Flow. J. Atm. Sci., 20, 130-141. https://doi.org/10.1175/1520-0469(1963)020<0130:DNF>2.0.CO;2

[2] Rössler, O. E. (1976). An Equation for Continuous Chaos. Physics Letters A, 57, 397-398. https://doi.org/10.1016/0375-9601(76)90101-8

[3] Chen, G. \& Ueta, T. (1999). Yet another Chaotic Attractor. Int. Journal of Bifurcation and Chaos, 9, 1465-1466.
https://doi.org/10.1142/S0218127499001024

[4] Pehlivan, I. \& Uyaroglu, Y. (2010). A New Chaotic Attractor from General Lorenz System Family and Its Electronic Experimental Implementation. Turkish Journal of Electrical Engineering and Computer Science, 18, 171184.

[5] Uyaroglu, Y. \& Pehlivan, I. (2010). Nonlinear Sprott94 Case A Chaotic Equation: Synchronization and Masking Communication Applications. Computers and Electrical Engineering, 36, 1093-1100. https://doi.org/10.1016/j.compeleceng.2010.04.002

[6] Pehlivan, I. \& Uyaroglu, Y. (2012). A New 3D Chaotic System with Golden Proportion Equilibria: Analysis and Electronic Circuit Realization. Computers and Electrical Engineering, 38(6), 1777-1784. https://doi.org/10.1016/j.compeleceng.2012.08.007

[7] Pehlivan, I. (2011). Four-Scroll Stellate New Chaotic System. Optoelectronics and Advanced Materials-Rapid Communications, 5(9), 1003-1006.

[8] Koyuncu, I., Ozcerit, A. T., \& Pehlivan, I. (2014). Implementation of FPGA-based Real Time Novel Chaotic Oscillator. Nonlinear Dynamics, 77(1-2), 49-59. https://doi.org/10.1007/s11071-014-1272-x

[9] Wei, Z., Zhang, W., Wang, Z., Yao, M. (2015). Hidden Attractors and Dynamical Behaviors in an Extended Rikitake System. International Journal of Bifurcation and Chaos, 25(2), 1550028 . https://doi.org/10.1142/S0218127415500285

[10] Wei, Z., Moroz, I. M., Wang, Z., Sprott, J. C., \& Kapitaniak, T. (2016). Dynamics at Infinity, Degenerate Hopf and Zero-Hopf Bifurcation for Kingni-Jafari System with Hidden Attractors. International Journal of Bifurcation and Chaos. 26(7), 1650125. https://doi.org/10.1142/S021812741650125X

[11] Wei, Z., Yu, P., Zhang, W., \& Yao, M. (2015). Study of Hidden Attractors, Multiple Limit Cycle from Hopf Bifurcation and Boundedness of Motion in the Generalized Hyperchaotic Rabinovich System. Nonlinear Dynamics, 82(1), 131-141. https://doi.org/10.1007/s11071-015-2144-8

[12] Birx, D. L. \& Pipenberg, S. J. (1992). Chaotic Oscillators and Complex Mapping Feed Forward Networks (CMFFN)s for Signal Detection in Noisy Environments. IEEE Int. Joint Conference on Neural Networks, 881-888. https://doi.org/10.1109/IJCNN.1992.226876

[13] Oppenheim, A. V., Wornell, G. W., Isabelle, S. H., \& Cuomo, K. M. (1992). Signal Processing in the Context of Chaotic Signals. Proceedings of the IEEE ICASSP / San Francisco, CA, IV, March 1992, 117-120. https://doi.org/10.1109/ICASSP.1992.226472

[14] Haykin, S. \& Xiao Bo, L. (1995). Detection of Signals in Chaos. Proceedings of the IEEE, 83, 95-122. https://doi.org/10.1109/5.362751

[15] Wang, G., Chen, D., Lin, J., \& Chen, X. (1999). The Application of Chaotic Oscillator to Weak Signal Detection. IEEE Transactions on Industrial Electronics, 46(2), 440-444. https://doi.org/10.1109/41.753783

[16] Wang, G. \& He, S. (2003). A Quantitative Study on Detection and Estimation of Weak Signal Using Chaotic Duffing Oscillator. IEEE Transaction on Circuits and Systems, 50(7), 945-953. https://doi.org/10.1109/TCSI.2003.812606

[17] Huiying, L., Zhiyu, C., Xiaopeng, Y., \& Ping, L. (2009). A Combined Method of Weak Signal Detection. IEEE Second Asia-Pacific Conference on Computation Intelligence and Industrial Applications, 106-109. https://doi.org/10.1109/PACIIA.2009.5406484

[18] Xuanchao, L. \& Xiaolong, L. (2011). Weak Signal Detection Research Based on Duffing Oscillator Used for Downhole Communication. Journal of Computers, 6(2), 359-367. 
[19] Jiang, F., Wang, X., Jin, J., \& Yang, D. (2010). The Application of Chaotic Duffing Oscillator to Ballistocardiograph Signal Detection. Proceedings of the Eighth World Congress on Intelligent Control and Automation / Jinan, China, July 2010, 6903-6906. https://doi.org/10.1109/WCICA.2010.5554236

[20] Jidong, J., Haihua, S., Teng, C., \& Shanming, L. (2010). Chaos Theory Apply in High Voltage Transmission Line Defect Detection. Proceedings of the Tenth IEEE Conference on Signal Processing, 17-21. https://doi.org/10.1109/ICOSP.2010.5656845

[21] Sun, Y., Zhan, X., Shi, J., \& Hu, Z. (2011). Based on Wavelet Denoising and Duffing Oscillator to Detect Weak Signal. Proceedings of the IEEE International Conference on Electronics, Communications and Control (ICECC), 1917-1920. https://doi.org/10.1109/ICECC.2011.6066665

[22] Yanchun, X., Xiaodong, Q., \& Chunling, Y. (2012). The Study of Novel Chaotic Oscillator Detecting Weak Signal Amplitude. Proceedings of the Seventh IEEE Conference on Industrial Electronics and Applications, 303-307.

[23] Gokyildirim, A., Uyaroglu, Y., \& Pehlivan, I. (2016). A Novel Chaotic Attractor and Its Weak Signal Detection Application. International Journal for Light and Electron Optics, 127(19), 7889-7895. https://doi.org/10.1016/j.ijleo.2016.05.150

[24] Li, Y., Yang, B. J., \& Shi, Y. W. (2003). Study on Chaotic Detection Method of $\mathrm{nV}$-level Sine Signal. Journal of Chine Institute of Communication, 24(4), 25-30.

[25] Chunyan, N. \& Yaowu, S. (2000). The Research of Chaotic Characteristic Identification Based on Weak Signal Detection. ACTA Metrological Sinica, 10(4), 308-313.

[26] Liu, D., Ren, H., Song, L., \& Li, H. (2005). Weak Signal Detection Based on Chaotic Oscillator. Proceedings of the IEEE Industry Applications Conference, Fourtieth IAS Annual Meeting, 2054-2058.

[27] Pehlivan, I., Moroz, I. M., \& Vaidyanathan, S. (2014). Analysis, Synchronization and Circuit Design of a Novel Butterfly Attractor. Journal of Sound and Vibration, 333(20), 5077-5096. https://doi.org/10.1016/j.jsv.2014.05.025

[28] Xie, T., Wei, X., \& Yu, R. (2010). Noise Immunity Analysis in External Excitation Chaotic Oscillator Detection System. International Conference on Intelligent System Design and Engineering Application, 1013-1016.

[29] Wei, Z., Wang, R, \& Liu, A. (2014). A New Finding of the Existence of Hidden Hyperchaotic Attractors with no Equilibria. Mathematics and Computers in Simulation, 100, 13-23. https://doi.org/10.1016/j.matcom.2014.01.001

[30] Wei, Z., Wang, R, \& Liu, A. (2014). Hidden Hyperchaotic Attractors in a Modified Lorenz-Stenflo System with Only One Stable Equilibrium. International Journal of Bifurcation and Chaos, 24(10), 1450127. https://doi.org/10.1142/S0218127414501272

[31] Sun, K., Liu, X., Zhu, C., \& Sprott, J. C. (2012). Hyperchaos and Hyperchaos Control of the Sinusoidally Forced Simplified Lorenz System. Nonlinear Dynamics, 69(3), 1383-1391.

[32] Zhao, D., Li, Y., Liu, X., \& Cao, Y. (2008). The Generation of a New Hyperchaos Based on Lorenz. Int. Conference on Intelligent Computation Technology and Automation, 10201024. https://doi.org/10.1109/ICICTA.2008.384

[33] Hu, W. \& Liu, Z. (2010). Study of Metal Detection Based on Chaotic Theory. Proceedings of the Eighth World Congress on Intelligent Control and Automation / Jinan, China, July 2010, 2309-2314.

[34] Honglei, Q., Xingli, S., \& Tian, J. (2010). Weak GPS Signal Detect Algorithm Based on Duffing Chaos System. Proceedings of the ICSP2, 2501-2508. https://doi.org/10.1109/ICOSP.2010.5656705
[35] Pehlivan, I. \& Wei, Z. (2012). Analysis, Nonlinear Control and Circuit Design of another Strange Chaotic System. Turkish Journal of Electrical Engineering and Computer Sciences, 20(2), 1229-1239.

[36] Cheng, F. \& Yan, Z. (2012). A New Method to Determine the Bifurcation Threshold Value of the Duffing Chaos Detection System. The Seventh International Conference on Computer Science and Education / Melbourne, Australia, July 2012, 1143-1146.

https://doi.org/10.1109/ICCSE.2012.6295267

\section{Contact information:}

\section{Abdullah GOKYILDIRIM}

(Corresponding author)

Sakarya University

Esentepe Campus

54187 Serdivan / Sakarya / Turkey

abdullah.gokyildirim@ogr.sakarya.edu.tr

\section{Yilmaz UYAROGLU}

Sakarya University

Esentepe Campus

54187 Serdivan / Sakarya / Turkey

uyaroglu@sakarya.edu.tr

\section{Ihsan PEHLIVAN}

Sakarya University

Esentepe Campus

54187 Serdivan / Sakarya / Turkey

ipehlivan@sakarya.edu.tr 